\title{
Differences in unpleasantness induced by experimental pressure pain between patients with fibromyalgia and healthy controls
}

\author{
Frank Petzke ${ }^{\mathrm{a}}$, Richard E. Harris ${ }^{\mathrm{b}}$, David A. Williams ${ }^{\mathrm{b}}$, \\ Daniel J. Clauw ${ }^{\text {, }}$, Richard H. Gracely ${ }^{\text {b,* }}$ \\ a Department of Anesthesiology of the University of Cologne, Germany \\ ${ }^{\mathrm{b}}$ Department of Medicine, Division of Rheumatology, University of Michigan, 3918 Taubman Center, \\ 1500 East Medical Center Drive, Ann Arbor, MI 48109-0358, USA
}

Received 20 January 2004; accepted 1 September 2004

Available online 8 October 2004

\begin{abstract}
Pain possesses both sensory and affective dimensions, which are highly correlated yet distinct. Comparison of these dimensions within experimental pain settings has resulted in the construct of relative unpleasantness. Relative unpleasantness is defined as the amount of affective unpleasantness elicited for a given sensory magnitude. The aim of this study was to determine the relationship between affective and sensory components of evoked pain in subjects with fibromyalgia (FM) and healthy controls. Here we show that patients with FM unexpectedly display less relative unpleasantness than healthy controls in response to random noxious pressure stimuli. Relative unpleasantness was not correlated with distress, anxiety, or depression, which were pronounced in the FM group. Clinical pain in patients with FM was perceived to be more unpleasant than the evoked pain stimuli. These results are consistent with the concept that chronic pain may reduce the relative unpleasantness of evoked pain sensations.

(C) 2004 Published by Elsevier Ltd on behalf of European Federation of Chapters of the International Association for the Study of Pain.
\end{abstract}

Keywords: Fibromyalgia; Pain; Unpleasantness; Sensory; Affective

\section{Introduction}

Pain is a sensation that is composed of distinct yet inter-related dimensions. Pain can be described in terms of its intensity or sensory qualities as well as its emotional or affective aspects, which are integral to the sensation. Primary affective pain is believed to occur over a short period of time and is related to the minute-by-minute appraisal of pain, whereas secondary affective pain involves both past and future long-term reflection of the sensation or condition. A final cognitive-evaluative \footnotetext{
0611.

* Corresponding author. Tel.: +1 734763 7518; fax: +1 734615

E-mail address: rgracely@med.umich.edu (R.H. Gracely).
}

dimension integrates both past experiences and judgments and exerts control over activity of both the sensory and affective systems (Melzack and Casey, 1968). This model has evolved from studies of both experimental and clinical pain (reviewed in: Fields, 1999; Gracely, 1999; Price, 2000).

Different clinical pain states can have distinct sensory and affective qualities. Price et al. (1987) observed that although women in labor and cancer patients experienced pain of similar sensory intensity, the affective component or unpleasantness of their pain was markedly different. Cancer patients reported more unpleasantness associated with their pain than did women in labor. Furthermore the unpleasantness that women in labor experienced was altered by their cognitive state. Accordingly, attention 
and other cognitive processes appear to modify pain perceptions (Malow, 1981; Miron et al., 1989; Keogh and Mansoor, 2001; Geisser et al., 2003).

Investigations of experimentally induced pain have also shown a divergence between pain dimensions (Gracely et al., 1979; Rainville et al., 1992) and this separation may be manifested in different cortical brain regions and modulated by different neurotransmitters. For example, the neural activity in the somatosensory cortex (S1) appears to correlate with pain intensity (Hofbauer et al., 2001) while activity in the anterior cingulate cortex (ACC) and other limbic structures may be the locus for unpleasantness (Rainville et al., 1997). Similarly, the affective and sensory components of pain are differentially modulated by pharmacologic therapies (Gracely et al., 1979). Given this divergence of sensory and affective aspects for both clinical and experimental pain, it is useful to define the measure of relative unpleasantness as a construct to assess this difference (Rainville et al., 1992). Relative unpleasantness can be thought of as "how much a given sensation bothers you" (Gracely, 1992).

Strong affective elements make the syndrome of fibromyalgia $(\mathrm{FM})$ ideal for examining differences in pain intensity and unpleasantness in both experimental and clinical settings. FM is defined by the presence of chronic, widespread pain, and the finding of generalized tenderness or increased pressure pain sensitivity on examination, as assessed by a tender point exam (Wolfe et al., 1990). A positive exam is defined by the presence of evoked pain at 11 out of 18 tender points when $4 \mathrm{~kg}$ of pressure is applied. In addition, FM patients commonly have high levels of psychological affective symptoms, such as anxiety and depression (Boissevain and McCain, 1991; Epstein et al., 1997), which could be an independent feature of the syndrome and/or a result from secondary aspects of this chronic condition. A complicating factor in FM studies is that the number of positive tender points has been shown to be influenced by an individual's subjective level of distress, so studies designed to isolate the affective component of the pain experience must control for this association (Wolfe, 1997; Petzke et al., 2003b).

More sophisticated experimental pain assessment methodologies may provide insight into the affective component of the pain experience in fibromyalgia. Most experimental pain studies in FM to date have used an "ascending" testing paradigm, such as manual tender point counts or dolorimetry, wherein the stimulus intensity is increased in a predictable manner (Bendtsen et al., 1997). Not surprisingly, subject and observer factors such as expectancy, affective state, or hypervigilance can influence pain report in these ascending paradigms (McDermid et al., 1996; Wolfe, 1997). Random testing paradigms, in which the next stimulus cannot be anticipated, have been shown to reduce these confounding factors (Petzke et al., 2003a,b), and are thought to min- imize the biasing effect of distress in determining a patient's degree of tenderness.

This study quantitatively describes the sensory intensity and affective unpleasantness components of evoked pressure pain in FM subjects and healthy controls (HC). Ratings were obtained from both ascending and random methods of stimulus presentation to evaluate the role of bias as outlined above. The specific focus was to determine if FM subjects had altered pain unpleasantness in response to evoked pressure stimuli relative to $\mathrm{HC}$ and if this difference would be correlated with measures of distress, dysfunction and clinical pain.

\section{Materials and methods}

\subsection{Subjects}

Patients under treatment at the Georgetown University Medical Center for an established diagnosis of FM were invited to participate in the study. Concurrent inflammatory rheumatic conditions or severe other medical conditions were criteria for exclusion. HC subjects were recruited through flyers and newspaper advertisements, compensated for their participation, and matched by age and sex to the patient population. Prior to the study, all participants read and signed an informed consent form. The Georgetown University Institutional Review Board approved the consent form and study protocols.

Because previous studies have shown differences in pain report for women at different stages in the menstrual cycle (Bajaj et al., 2001, 2002; Riley et al., 1999), all female participants were screened for menstrual status on the day of pain testing. They were asked about menstrual history, average cycle length, last menstrual period, and use of medications (birth control pill, hormone replacement therapy). Cycle stages were grouped into menstruation, follicular (including midcycle), luteal (including premenstrual), and postmenopausal (including perimenopausal women, and subjects with hysterectomy).

For patients with FM the presence of chronic widespread pain was confirmed, and a manual tender point count performed. Only patients that satisfied the ACR criteria (Wolfe et al., 1990) of at least 11 of 18 positive tender points were included in the analysis. All control subjects underwent a tender point examination and were excluded if they had a tender point count of 11 or greater or a history of any current or chronic pain of greater than one-week duration.

\subsection{Psychophysical testing - overview}

Upon arrival to the research center all subjects were shown and oriented to the experimental pain-testing environment. During that time the consent form was signed 
and remaining questions answered, the menstruation history obtained, and subjects were asked about current medications and their intake in the last $24 \mathrm{~h}$. Patients participating in pain testing were allowed to continue their regular medication. However, they were advised not to take any analgesics for $24 \mathrm{~h}$ prior to the testing session. Healthy controls were also asked not to take any analgesic medication $24 \mathrm{~h}$ prior to the pain testing.

The pain testing equipment was demonstrated and explained using a "scripted" text. A few discrete pressure stimuli were applied to familiarize subjects with the procedure. The instructions for the different tests also followed a standardized script and additional information and explanations were provided if required. The sequence of testing was the same for all subjects.

\subsection{Pressure pain testing}

A dolorimeter exam at the 18 defined tender points, and four control points (the bilateral thumbs and anterior tibial muscles) was performed on all subjects (Wolfe et al., 1990) using a $3.14 \mathrm{~cm}^{2}$ footplate size. Pressure was increased at a rate of $1 \mathrm{~kg} / \mathrm{s}$ and subjects were instructed to indicate when they first perceived pain. If no pain response was elicited up to $12 \mathrm{~kg}$ of pressure, this value was recorded as the pain threshold. The average pressure pain threshold for all 18 tender point sites was calculated and expressed as $\mathrm{kg} / 3.14 \mathrm{~cm}^{2}$.

Discrete pressure stimuli were applied using a remote stimulation device to eliminate any direct examiner/subject interaction. The apparatus induced pressure with a hydraulic system in which a $1 \mathrm{~cm}^{2}$ hard rubber circular probe was pressed against the left or right thumbnail (see below). The thumbnail was chosen as it has been shown to be more sensitive to pressure in FM subjects than in HC (Petzke et al., 2001), and remains a "neutral" point for patients with FM compared to a typical tender point. The stimulator was positioned over the thumb by a plastic housing and the hydraulic system activated by calibrated weights placed on a moveable table. Valves controlled stimulus timing. The combination of valves and calibrated weights produced controlled, repeatable stimulation that approached a rectangular waveform.

To compare the effect of stimulus presentation on unpleasantness and intensity ratings, both an ascending and a random series were performed on each subject.

\subsubsection{Discrete ascending pressure}

For the ascending series (ASC), discrete stimuli of $5 \mathrm{~s}$ duration were applied to the right thumbnail. Initial stimulation pressure was $0.45 \mathrm{~kg}$ and stimulation pressure was increased in $0.45 \mathrm{~kg}$ increments up to either a subject's level of pain tolerance or a maximum of 9.1 $\mathrm{kg}$. Subjects used two 21-box numerical descriptor scales to rate the intensity and then the unpleasantness of sensations evoked by each stimulus (see Fig. 1). After sub- jects had rated a stimulus with a pain intensity of greater than 10 (mild to moderate pain), they were asked if they wished to continue after each succeeding stimulus. Interstimulus interval was $30 \mathrm{~s}$.

\subsubsection{Discrete random pressure}

For the random series (RAN), subjects were instructed that they would receive a different series of stimuli within the range of the previous ascending series. If tolerated, seven stimuli $(0.45,0.91,1.36,1.82,2.73$, $3.64,4.54 \mathrm{~kg}$ ) were twice presented in random order to the left thumb and ratings of sensory intensity and unpleasantness were obtained using the 21-box scales. Four subjects with FM received less than the seven stimuli ( 1 only four stimuli, and 3 only 6 stimuli). In both patients and controls this weight distribution resulted in at least three values between pain threshold and tolerance. Inter-stimulus interval was $30 \mathrm{~s}$.

\subsection{Pain intensity and unpleasantness estimation}

Pain intensity and unpleasantness ratings in response to discrete stimuli were recorded on two separate 21-box numerical descriptor scales (Fig. 1) using standardized instructions, similar to Price et al. (1984). These scales

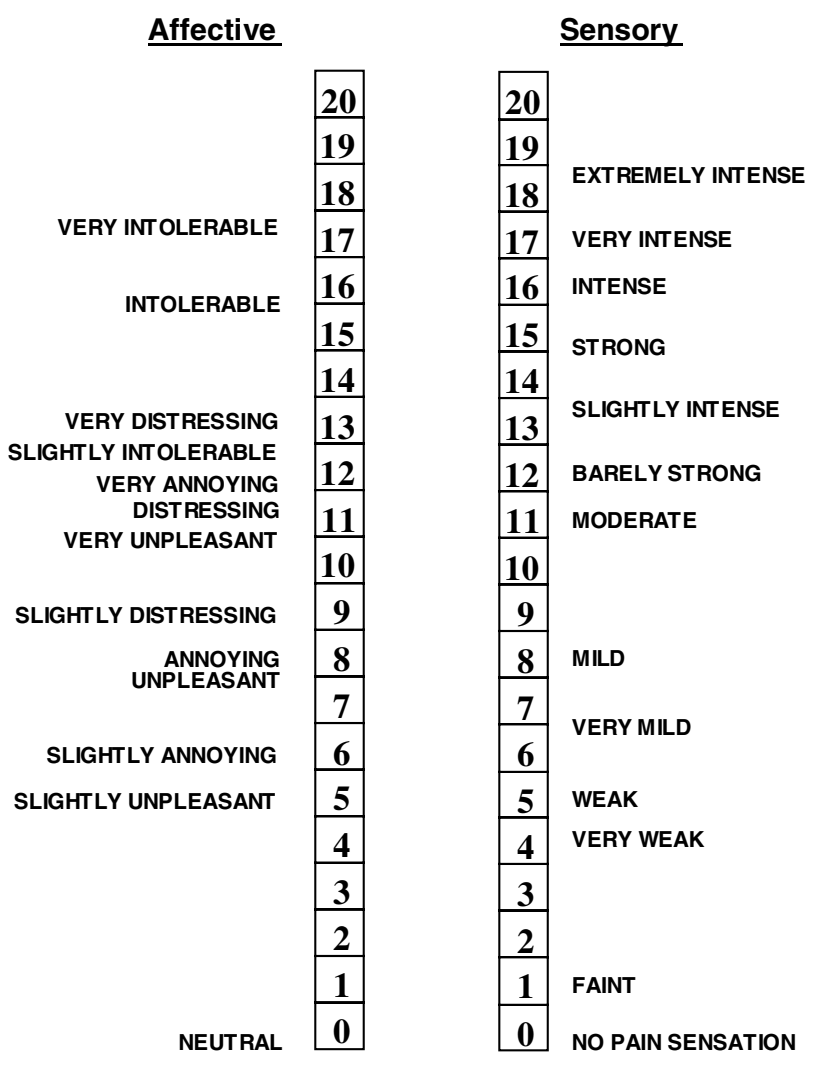

Fig. 1. Affective and sensory numerical descriptor scales used to measure evoked pain dimensions of affective unpleasantness and sensory intensity. 
had been constructed from previously quantified verbal descriptors (Gracely et al., 1978a,b, 1979). They posses logarithmic properties with regard to the spacing of the descriptors, and had been shown to be sensitive in other studies (Eliav and Gracely, 1998; Sternberg et al., 1998; Gracely et al., 2002; Lembo et al., 2000; Petzke et al., 2003a).

In addition to the two 21-box scales described above, experimental pain was also assessed with the Short Form of the McGill pain questionnaire (MPQ:SF). Subjects were asked to rate the worst pain experienced during the two prior testing sessions (ASC; RAN). Thus the subjective ratings were not in response to a specific stimulus but reflected the retrospective perception and experience during the two evoked pain paradigms.

\subsection{Questionnaires}

\subsubsection{Functional status}

The SF-36, is a brief, well-established, self-administered patient questionnaire for the assessment of health status (Ware et al., 2000). The SF-36 measures eight domains of health status, and summary physical (PCS) and mental health (MCS) scales can be calculated by combining and weighting the various individual scales. These summary scales are standardized to have a mean $=50, \mathrm{SD}=10$ in the general US population (Ware and Kosinski, 2001).

\subsubsection{Depressive symptoms}

Neurovegetative and cognitive symptoms of depression were evaluated by the Beck Depression Inventory (BDI), a 21-item measure of the severity of current depressive symptoms that has been validated for use in rheumatic diseases (Burckhardt et al., 1994).

\subsubsection{Distress}

The Brief Symptom Inventory (BSI) was used to obtain an indicator of distress (Derogatis and Spencer, 1983). This 51 -item instrument contains nine sub-scales and a Global Severity Index (GSI), which was used as a general measure for distress; the anxiety sub-scale was used to measure an individual's anxiety.

\subsubsection{Clinical pain}

Clinical pain was assessed by the Short Form of the McGill pain questionnaire (MPQ:SF). This 15-item inventory yields both a sensory and affective subscale (Melzack, 1987). Scores were expressed as \% of the maximum possible score for each dimension (Stohler and Kowalski, 1999).

\subsection{Statistical analysis}

Statistical analyses were performed in several stages: (1) Descriptive statistics defining similarities and differ- ences between the FM and healthy control groups, (2) Analyses comparing pain intensity and unpleasantness between the FM and $\mathrm{HC}$ groups in the ASC and RAN series of tests, (3) Analyses comparing the relative unpleasantness between the FM and HC groups, (4) Analyses addressing the influence of differences in mean pain intensity between the two groups on the relative unpleasantness, (4) Analyses exploring associations between relative unpleasantness and affective symptoms, and (5) the relative unpleasantness of clinical compared to evoked pain in patients with FM.

\subsubsection{Comparison of pain intensity and unpleasantness}

To compare responses in the discrete pressure testing paradigms (ASC, RAN), the areas under the stimulus response curve (AUC) for pain intensity and unpleasantness for the $0.45-4.5 \mathrm{~kg}$ stimulus range were calculated for both groups. Since the curve was defined as a set of stimulation pressures $\left(x_{i}\right)$ and corresponding pain ratings $\left(y_{i}\right)$, the AUC was calculated as follows: $\mathrm{AUC}=\sum\left(\left(\left(y_{(i+1)}+y_{i}\right) / 2\right) \times\left(x_{(i+1)}-x_{i}\right)\right)$. The sum included all available data points in a given stimulus range. In subjects who could not complete the whole testing range the highest pain intensity or unpleasantness rating was substituted for the missing values (only six values in four FM subjects).

\subsubsection{Relative unpleasantness}

Relative unpleasantness is typically defined as the ratio of unpleasantness to intensity ratings (unpleasantness/intensity; (Rainville et al., 1992)). Since the box scales we used possess logarithmic properties (Gracely et al., 1978a), relative unpleasantness was calculated as the difference of unpleasantness minus intensity, which corresponds to a ratio in arithmetic units. We calculated the mean relative unpleasantness as the AUC for unpleasantness ratings minus the AUC for intensity ratings.

Relative unpleasantness for clinical and evoked pain ratings with the MPQ:SF in patients with FM was also calculated. The respective MPQ:SF \% scores were used as follows: \%affective score $/ \%$ sensory score.

\subsubsection{Mean perceived pain intensity and range restriction}

The restriction of stimulus intensity to a range from 0.45 to 4.5 in ASC and RAN was necessary to allow the comparison of the average group responses. With respect to the relationship between ratings of pain intensity and unpleasantness in the two groups, this range restriction could result in a scaling bias due to the group difference in pain sensitivity, with FM patients typically using higher scale values than HC. Therefore the mean perceived pain intensity was calculated for ASC and RAN and correlated with the respective relative unpleasantness. 
To assess the use of the unpleasantness and intensity scales across the whole individual rating range of the AUCs, the relative unpleasantness and the mean perceived pain intensity were also calculated for all available responses in the ascending paradigm up to individual tolerance or the stimulus maximum of $9.1 \mathrm{~kg}$ (ASCTOL) in both groups.

\subsection{Statistical procedures}

$t$-Tests and $\chi$-square tests were used to characterize the demographic (e.g. age, sex,), clinical pain characteristics (menstrual status, disease duration, MPQ:SF pain scores, tender point and dolorimetry scores), and affective measures (BDI, BSI). ANOVAs were used to evaluate whether unpleasantness or relative unpleasantness differed between groups and across pain-evoking paradigms. Correlational analyses were used to explore associative relationships between relative unpleasantness and mean perceived pain intensity, and affective variables. All data are expressed as mean \pm SEM unless stated otherwise. Data analysis was performed with SPSS 11.0 and MS Excel.

\section{Results}

\subsection{Subjects}

The 43 clinic patients included in the study fulfilled the ACR criteria for FM on the day of testing. Subjects were age and gender matched with 28 healthy control subjects (Table 1). The observed slight difference in age was not statistically significant $(p=0.26)$ nor was the proportion of males to females $(p=0.75)$. Distribution of menstrual status in the two groups was similar: $62 \%$ of patients and $50 \%$ of controls were postmenopausal; cycle stages in the remaining subjects were evenly distributed between the two groups and not statistically significantly different $(\chi$-square $p=0.29)$. Not surprisingly, FM subjects displayed statistically significant greater clinical pain scores (MPQ:SF), depression, distress, and tenderness compared to controls. Only four of the healthy controls had more than four positive tender points on manual palpation.

\subsection{Pressure thresholds - dolorimetry on thumb and tender points}

As expected, all pressure pain thresholds were significantly higher in the control subjects than in the FM patients (Table $1 ; 8.5 \pm 0.35$ vs. $4.5 \pm 0.2 \mathrm{~kg} / \mathrm{cm}^{2}$ for tender points; $10.0 \pm 0.4$ vs. $6.1 \pm 0.4 \mathrm{~kg} / \mathrm{cm}^{2}$ for right thumb; $10.6 \pm 0.4$ vs. $6.1 \pm 0.4 \mathrm{~kg} / \mathrm{cm}^{2}$ for left thumb; all $p<0.0001)$. Within the groups, measures at the thumbs and tender points were highly inter-correlated; more so in the patient group $(r=0.73-0.93$; all $p<0.0001)$ than in the controls $(r=0.54-0.73$; all $p<0.003)$ possibly due to range restriction in the latter group.

\subsection{Unpleasantness and pain intensity}

\subsection{1. $A S C$}

Patients with FM had higher AUC summary ratings than healthy controls for both unpleasantness and pain intensity in the ascending paradigm (Fig. 2(a)). A $2 \times 2$ ANOVA of group $\mathrm{X}$ pain dimension revealed significant main effects for group $(\mathrm{FM}>\mathrm{HC}, F[1,69]=30.3$, $p<0.001$ ) and dimension (Unpleasantness > Intensity, $F[1,69]=5.1, p<0.03)$, but no significant interaction $(F[1,69]=0.9, p=0.35)$. This indicated that the difference between unpleasantness and intensity within both groups was similar.

\subsection{2. $R A N$}

Similar to the ASC paradigm, patients with FM had an increased AUC for unpleasantness and intensity compared to HC for RAN (Fig. 2(b)). A $2 \times 2$ ANOVA

Table 1

Clinical characteristics of the two subject groups: age, male/female ratio, average subject questionnaire responses, and standard pain measures $($ mean \pm SDV)

\begin{tabular}{llll}
\hline & Fibromyalgia patients, $N=43$ & Healthy controls, $N=28$ & $p$ \\
\hline Age (years) & $49.7 \pm 11.7$ & $46.8 \pm 9.5$ & n.s. \\
Male (N)/Female (N) & $4 / 39$ & $2 / 26$ & n.s. \\
Disease duration (years) & $10.5 \pm 8.0$ & - & $1.1 \pm 0.4$ \\
Regional pain score & $40.9 \pm 16.8$ & $0.4 \pm 0.1$ & 0.0001 \\
MPQ:SF - total score & $18.3 \pm 8.2$ & $0.4 \pm 0.1$ & 0.0001 \\
MPQ:SF - sensory score & $14.7 \pm 6.1$ & $0.0 \pm 0.0$ & 0.0001 \\
MPQ:SF - affective score & $3.8 \pm 3.1$ & $46.2 \pm 1.9$ & 0.0001 \\
BSI Global Severity Index & $63.8 \pm 1.4$ & $2.7 \pm 0.6$ & 0.0001 \\
BDI & $15.3 \pm 10.1$ & $2.1 \pm 0.57$ & 0.0001 \\
Manual tender point count & $14.3 \pm 0.34$ & {$[0-8]$} & 0.0001 \\
(range) & {$[11-18]$} & $8.55 \pm 0.35$ & \\
Dolorimetry pain threshold $\left(\mathrm{kg} / 3.14 \mathrm{~cm}^{2}\right)$ & $4.47 \pm 0.22$ & & 0.0001 \\
\hline
\end{tabular}




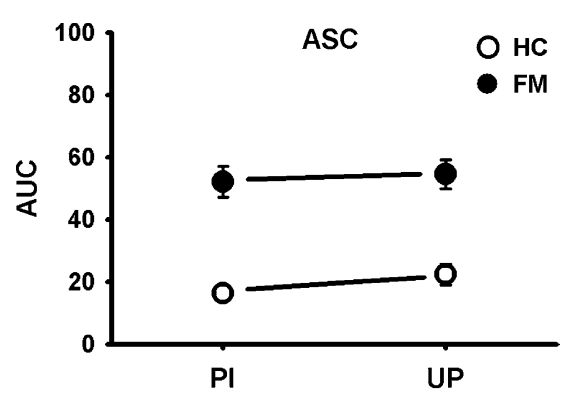

(a)

Dimension

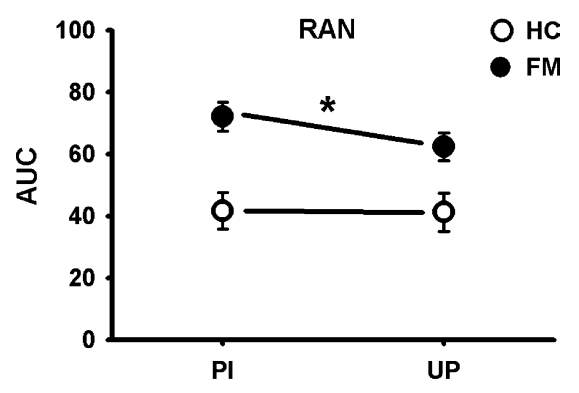

(b)

\section{Dimension}

Fig. 2. Pain intensity (PI) and unpleasantness (UP) ratings for FM and $\mathrm{HC}$ expressed as area under the curve (AUC) \pm 1 SEM. (a) ASC The FM group displayed greater PI and UP compared to HC $(\mathrm{PI}=52.1 \pm 4.99 \quad \mathrm{FM}, \quad 16.3 \pm 2.36 \quad \mathrm{HC} ; \quad \mathrm{UP}=54.5 \pm 4.70 \quad \mathrm{FM}$, $22.3 \pm 3.28 \mathrm{HC}$ ), however there was no significant interaction. (b) RAN - Similar to the ASC paradigm, the FM group had greater PI and UP compared to $\mathrm{HC}(\mathrm{PI}=72.04 \pm 4.62 \mathrm{FM}, 41.6 \pm 5.91 \mathrm{HC}$; $\mathrm{UP}=62.3 \pm 4.56 \mathrm{FM}, 41.2 \pm 6.17 \mathrm{HC})$. However within groups, FM subjects displayed less UP than PI as compared to HC (* significant interaction).

of RAN group $\mathrm{X}$ pain dimension revealed significant main effects for group $(\mathrm{FM}>\mathrm{HC}, F[1,69]=12.5$, $p<0.001)$ and pain dimension, however the dimension effect was reversed in direction (unpleasantness $<$ intensity, $F[1,69]=9.3, p<0.003$ ) as compared to the ASC paradigm. A significant group $\mathrm{X}$ dimension interaction $(F[1,69]=7.9, p=0.007)$ was detected indicating that the difference between unpleasantness and intensity ratings within the FM group differed from the $\mathrm{HC}$ group.

\subsection{Relative unpleasantness}

The difference in degree and direction of the interaction between group $X$ pain dimensions seen in Fig. 2 is explained by Fig. 3, which shows the relative unpleasantness for ASC and RAN. Patients with FM displayed generally less relative unpleasantness and this difference was significant and pronounced in the random paradigms $(p<0.01)$.

We compared relative unpleasantness in ASC and RAN using a $2 \times 2$ ANOVA to analyze the effect of pain testing methodology. A significant main effect for group was observed $(\mathrm{FM}<\mathrm{HC}: F[1,69]=4.0, p<0.05)$. The effect of method (ASC $>$ RAN) was also highly signifi-

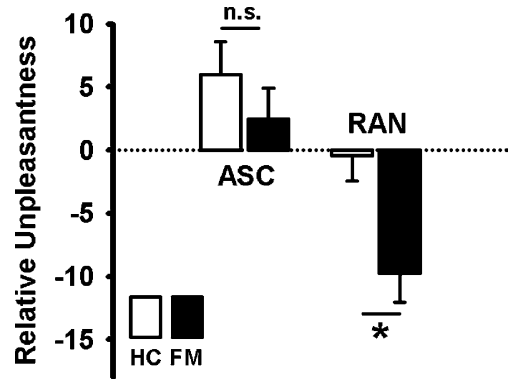

Fig. 3. Relative unpleasantness (AUC for UP minus AUC for PI) \pm 1 SEM in the two paradigms: For ASC a student's $t$-test revealed no significant difference $(p=0.35)$ between FM $(2.44 \pm 2.48)$ and HC $(5.96 \pm 2.62)$. However for RAN, relative unpleasantness was significantly less for $\mathrm{FM}$ as compared to $\mathrm{HC}$ (RAN: ${ }^{*} p<0.007$, $-9.75 \pm 2.33 \mathrm{FM},-0.41 \pm 2.05 \mathrm{HC}$ ).

cant $(F[1,69]=37.8, \quad p<0.0001)$, while the groupmethod interaction approached significance $(F[1,69]=$ $3.7, p=0.06$ ). These findings indicate (1) that both patients with FM and $\mathrm{HC}$ described equally intense pain sensations as more unpleasant in the ascending paradigm compared to the less predictable random paradigm, (2) that this difference between paradigms is more pronounced in patients with FM and (3) that patients with FM report generally less relative unpleasantness than HC.

The above data indicated that with random stimulus presentation, despite overall higher pain and unpleasantness ratings than HC, FM patients had relatively less unpleasantness compared to HC. This can also be visualized by plotting the mean unpleasantness score versus the mean pain intensity score for all pressures eliciting a painful sensation (mean pain intensity score $>0.5$ representing faint pain) in the ASC (Fig. 4(a)) and RAN paradigms (Fig. 4(b)). Mean scores in the ascending paradigm showed a similar linear pattern in both groups, with the FM curve shifted towards higher values. However, FM mean scores in the random paradigm consistently trended toward lower values along the affective dimension, suggesting an additional parallel downward shift in relative unpleasantness over the whole stimulus-response curve.

Affective and sensory sub-scale scores of evoked experimental pain were also compared using the MPQ:SF. Similar to the above findings, subjects with FM reported relatively less evoked affective pain as measured by the MPQ:SF (Fig. 5: Evoked pain in $\mathrm{HC}$ and FM). Although the sensory scores were significantly higher $(\mathrm{FM}>\mathrm{HC} ; p<0.05)$, the affective scores were not statistically different $(p=0.29)$. Thus, a combination of a different scale and a retrospective assessment also showed that the relative unpleasantness of evoked pain was again less for the FM subjects (i.e. identical unpleasantness for an increased sensory intensity). 

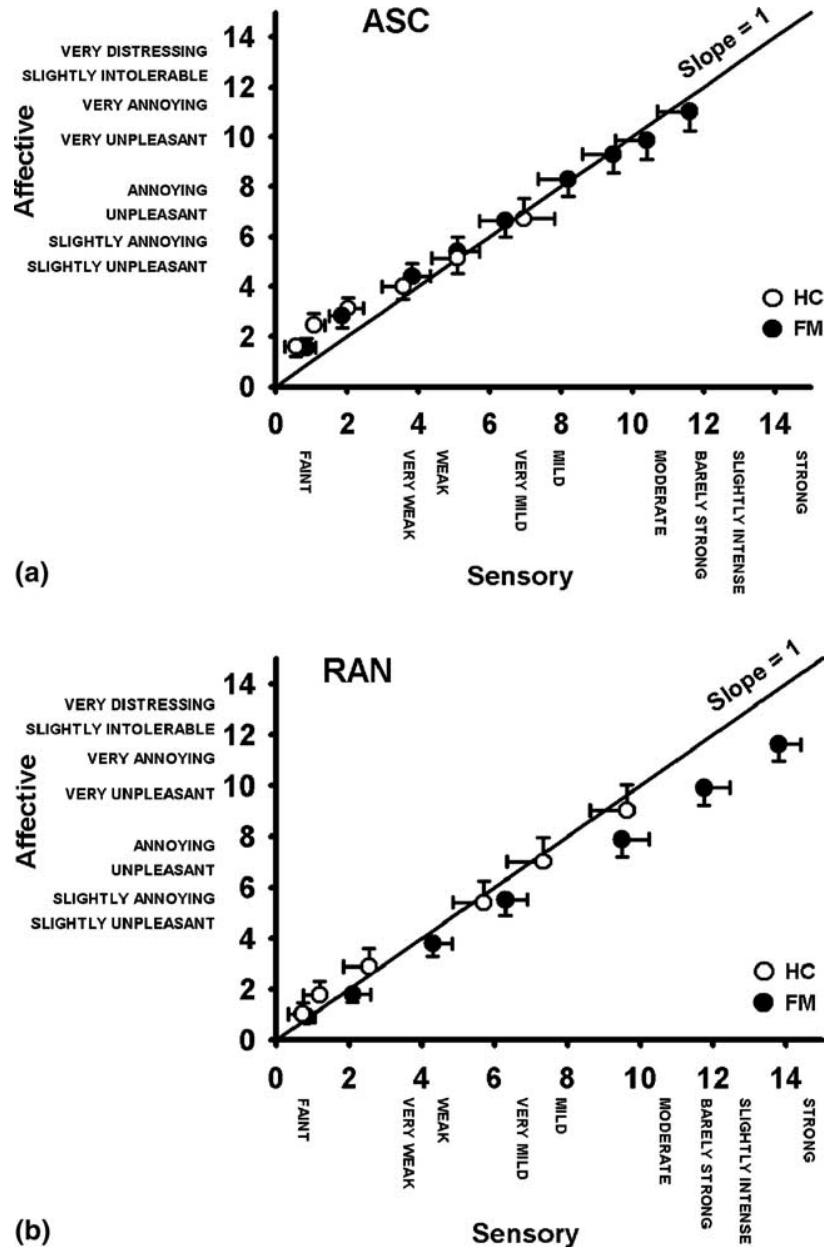

Fig. 4. Affective versus sensory plots: (a) ASC - 21-box scale mean values \pm 1 SEM for affective and sensory ratings for $0.91,1.36,1.82$, $2.27,2.73,3.18,3.64,4.09$, and $4.54 \mathrm{~kg}$ weights in FM and 2.27, 2.73, $3.18,3.64,4.09$, and $4.54 \mathrm{~kg}$ weights in $\mathrm{HC}$, representing stimuli inducing an average pain intensity rating $>0.5$ (faint pain). FM subjects tend to greater affective and sensory scores but show a similar stimulus-response curve. (b) RAN - 21-box scale mean values \pm 1 SEM for affective and sensory ratings in both groups for 0.45 (not in HC), $0.91,1.36,1.82,2.73,3.64$, and $4.54 \mathrm{~kg}$ weights, representing stimuli inducing an average pain intensity rating $>0.5$ (faint pain). FM subject means tended toward lower affective and greater sensory scores resulting in a parallel downward shift of the stimulus-response curve.

\subsection{Correlation of relative unpleasantness with mean perceived pain intensity}

A significant effect of the pain testing methods on relative unpleasantness was found for both groups, with higher relative unpleasantness ratings in the ascending series (Fig. 3). However, overall ratings of unpleasantness and pain intensity were higher in the random than in the ascending paradigms for both groups (Figs. 2 and 4(a) and (b)). Therefore we examined the relationship between mean perceived pain intensity and relative unpleasantness for both FM and HC subjects (Table 2). The relative unpleasantness for patients with FM

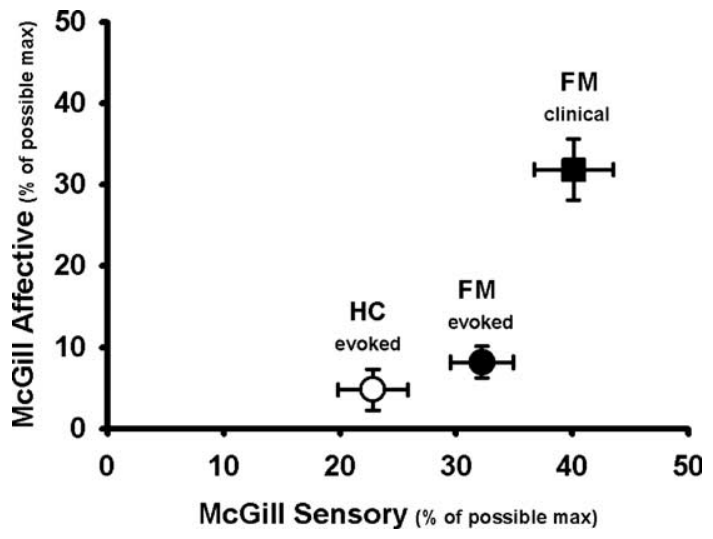

Fig. 5. Retrospective ratings of experimental pressure pain (for $\mathrm{HC}$ and FM) and clinical pain (for FM only) using the MPQ:SF affective and sensory sub-scales, expressed as \% of possible maximum score. FM and $\mathrm{HC}$ evoked pain: The sensory mean was greater in FM than $\mathrm{HC}(p<0.05: 32.2 \pm 2.72 \mathrm{FM}, 22.8 \pm 2.98 \mathrm{HC})$, however the affective scores were not significantly different $(p=0.29$ : $8.14 \pm 1.95$ FM, $4.76 \pm 2.55 \mathrm{HC}$ ). FM evoked and clinical pain: FM patients reported more sensory and affective clinical pain, with more relative unpleasantness compared to the evoked pain.

correlated negatively with perceived pain intensity in the ASC and RAN paradigm, while HC displayed a similar but non-significant trend. This negative correlation, however, was significant for both groups in ASC-TOL when the entire stimulus range that was delivered during the ascending paradigm was evaluated. This relationship is also evident in Figs. 4(a) and (b) if the position of pressure stimuli in both groups is compared to the bisecting line (indicating equal pain intensity and unpleasantness). In Fig. 4(a), stimuli inducing higher pain intensity ratings generally lie below this line (indicating less relative unpleasantness) while stimuli inducing lower pain intensity are positioned above (indicating more relative unpleasantness). In Fig. 4(b) the same relationship is seen, however, patients with FM reported generally less unpleasantness resulting in a parallel and downward curve shift (see above).

The combination of the restriction of the stimulus range in the ASC and RAN paradigms and the negative correlation of relative unpleasantness with pain intensity may have contributed to the differences in relative unpleasantness observed between patients with FM and $\mathrm{HC}$, but it fails to explain the increasing group differences in relative unpleasantness in the random paradigms with overall higher pain intensity and unpleasantness ratings.

\subsection{Correlation with psychometric measures}

We examined the interaction of relative unpleasantness with the psychological constituents of each subject to determine if psychological parameters could account for the differences observed above. The 
Table 2

Correlation between relative unpleasantness and mean perceived pain intensity in the three paradigms, and ASC-TOL for both patients with FM and $\mathrm{HC}$

\begin{tabular}{|c|c|c|c|c|c|c|c|}
\hline \multirow{3}{*}{$\begin{array}{l}\text { Mean perceived } \\
\text { Pain intensity }\end{array}$} & & \multicolumn{6}{|c|}{ Relative unpleasantness } \\
\hline & & \multicolumn{2}{|l|}{ ASC } & \multicolumn{2}{|l|}{ RAN } & \multicolumn{2}{|c|}{ ASC-TOL } \\
\hline & & $\mathrm{HC}$ & $\mathrm{FM}$ & $\mathrm{HC}$ & FM & $\mathrm{HC}$ & FM \\
\hline \multirow[t]{2}{*}{$\overline{\mathrm{ASC}}$} & $\mathrm{r}$ & -0.11 & -0.39 & - & - & & \\
\hline & $\mathrm{p}$ & 0.56 & 0.009 & - & - & & \\
\hline \multirow[t]{2}{*}{ RAN } & $\mathrm{r}$ & - & - & -0.23 & -0.26 & & \\
\hline & $\mathrm{p}$ & - & - & 0.24 & 0.09 & & \\
\hline \multirow[t]{2}{*}{ ASC-TOL } & $\mathrm{r}$ & & & & & -0.37 & -0.35 \\
\hline & $\mathrm{p}$ & & & & & 0.05 & 0.02 \\
\hline
\end{tabular}

Table 3

Correlation between relative unpleasantness in the three paradigms, ASC-TOL and psychometric measures of distress (BSI-GSI), anxiety (BSI-subscale), depression (BDI), and clinical pain (MPQ:SF affective and sensory)

\begin{tabular}{|c|c|c|c|c|c|}
\hline & & \multicolumn{4}{|c|}{ Relative unpleasantness } \\
\hline & & \multicolumn{2}{|l|}{ ASC } & \multicolumn{2}{|l|}{ RAN } \\
\hline & & $\mathrm{HC}$ & FM & $\mathrm{HC}$ & FM \\
\hline \multirow[t]{2}{*}{ BSI-Anxiety } & $\mathrm{r}$ & -0.18 & -0.14 & 0.1 & 0.01 \\
\hline & $\mathrm{p}$ & 0.37 & 0.37 & 0.62 & 0.96 \\
\hline \multirow[t]{2}{*}{ BSI-GSI } & $\mathrm{r}$ & 0.05 & 0.1 & 0.13 & 0.18 \\
\hline & $\mathrm{p}$ & 0.81 & 0.55 & 0.51 & 0.26 \\
\hline \multirow[t]{2}{*}{ BDI } & $\mathrm{r}$ & 0.06 & 0.03 & -0.10 & 0.17 \\
\hline & $\mathrm{p}$ & 0.76 & 0.85 & 0.63 & 0.29 \\
\hline \multirow[t]{2}{*}{ MPQ:SF - Total score } & $\mathrm{r}$ & 0.03 & 0.002 & -0.2 & 0.05 \\
\hline & $\mathrm{p}$ & 0.87 & 0.98 & 0.32 & 0.77 \\
\hline \multirow{2}{*}{ MPQ:SF - Sensory score } & $\mathrm{r}$ & 0.03 & -0.01 & -0.2 & -0.02 \\
\hline & $\mathrm{p}$ & 0.87 & 0.98 & 0.32 & 0.92 \\
\hline \multirow[t]{2}{*}{ MPQ:SF - Affective score } & $\mathrm{r}$ & n.a. ${ }^{a}$ & 0.01 & n.a. ${ }^{a}$ & -0.01 \\
\hline & $\mathrm{p}$ & & 0.52 & & 0.97 \\
\hline
\end{tabular}

${ }^{a}$ All HC had a MPQ:SF affective score of 0.

correlation coefficients for the relationships between relative unpleasantness in the ASC and RAN paradigms and psychometric measures for anxiety, distress, depression, and clinical pain are shown in Table 3. None of the correlations were significant in either group.

\subsection{Evoked and clinical pain}

The MPQ:SF provides the opportunity to compare pain dimensions in both clinical and evoked settings within the FM group (Fig. 5). For the FM subjects alone a $2 \times 2$ ANOVA showed significant main effects for type of pain (clinical $>$ experimental, $F[1,42]=76.4, \quad p<$ 0.0001 ), pain dimension (sensory $>$ affective, $F[1,42]=$ $138.4, p<0.001)$, and a highly significant interaction $(F[1,42]=68.0, \quad p<0.0001)$. Relative unpleasantness was $0.83 \pm 0.09$ for clinical but only $0.23 \pm 0.05$ for evoked pain $(p<0.0001)$. These results indicated that clinical pain was more unpleasant than experimental pain of a given sensory intensity.

\section{Discussion}

This study examined two dimensions of pain, intensity and unpleasantness, in both FM and HC subjects. The primary finding from this investigation of noxious pressure was that patients with FM reported greater pain intensity but less relative unpleasantness as compared to healthy controls. These results suggest that although FM patients experience clinical pain on a regular basis, they are less bothered by pain in experimental settings. This difference may be explained by multiple factors, which may not be mutually exclusive.

\subsection{Why do FM subjects display relatively less unpleasantness?}

FM patients experience pain of a significant magnitude for a prolonged duration of time. The mean duration of the disease for patients in this study was 10.5 years. Therefore the stimuli used in these experiments might be perceived as relatively less unpleasant due to 
the fact that FM patients are simply more familiar with painful sensations than controls. A similar explanation could be that because the FM patients have already experienced clinical pain unpleasantness of significant magnitude, they compare the evoked stimuli to their greater clinical pain. Indeed in this study the FM subjects using the MPQ:SF reported their clinical pain as more unpleasant than evoked pain of similar intensity (Fig. 5). This effect seen in clinical pain subjects was predicted by Rollman and was termed adaptation (Rollman, 1979, 1983). He reasoned that subjects use their clinical pain as a reference point for pain in experimental settings. In contrast, this reference framework may be missing in the control subjects.

In addition to an adaptation effect operating during the evaluation and rating of pain experience, it is also possible that the relative unpleasantness observed with FM may reflect modulation by intrinsic mechanisms activated by persistent pain. For example, several studies have found that endogenous analgesic systems can be activated in healthy controls but not in patients with FM (Kosek et al., 1996; Lautenbacher and Rollman, 1997; Staud et al., 2003). Although often interpreted as a disorder in intrinsic analgesia, this result is also consistent with a state in which the persistent pain of FM results in maximal tonic activation of endogenous analgesic systems (Gracely et al., 2003), and that the reduction in relative unpleasantness observed with FM reflects preferential modulation of pain unpleasantness. Neither the adaptation nor this modulation mechanism needs to be mutually exclusive and both mechanisms could contribute to our results.

\subsection{Why does random presentation differ from ascending?}

An interesting finding in this study was that differences in relative unpleasantness were detected only when the painful stimuli were presented in a random fashion. When the stimuli were presented in a predictable ascending fashion there was no group $\mathrm{X}$ paindimension interaction. Some of this difference might be explained by the generally higher pain ratings from FM patients for a given stimulus intensity and the trend to less relative unpleasantness with higher pain ratings in both groups (Table 2 and Fig. 4(a) and (b)). But the similar linear pattern of mean pain intensity and unpleasantness ratings of ascending pressure stimuli present in both groups (Fig. 4(a)), is contrasted by a parallel downward shift in unpleasantness ratings in patients with fibromyalgia in response to randomly presented stimuli (Fig. 4(b)). Thus, mechanisms other than the properties and range restriction of the pain scales likely mediate the observed difference in relative unpleasantness.

The cognitive context of the random and ascending paradigms are clearly different. In the ascending para- digm, subjects know that the intensity of the next stimulus will be just slightly greater than the intensity of the preceding stimulus. In contrast, the unpredictable quality of the random presentation results in a state of uncertainty that may lead to exaggerated responses. Unpredictable painful stimuli have been previously shown to be more unpleasant than anticipated painful stimuli (Price et al., 1980) and even non-painful stimuli appear more unpleasant when presented in a random fashion (Sawamoto et al., 2000). Our finding that ratings of both intensity and unpleasantness were higher in the random than the ascending series in both groups (Fig. 2) is consistent with these previous results. At this point it is important to realize that patients with FM were even less inclined than $\mathrm{HC}$ to respond in an exaggerated fashion, showing less relative unpleasantness in the random paradigm and that this relative unpleasantness was not correlated to any of the psychosocial or clinical pain measures.

It is also conceivable that the difference between the ascending and random paradigms reflects the effects of task demands in the ascending methods. Subjects know that the stimulus intensity increases on successive trials, and thus likely adapt a response behavior in which successive responses also increase. This potentially produces results that are automatically monotonic with stimulus intensity. Subjects can produce such data without even attending to the stimulus (Gracely et al., 2003). This is an example of a "stimulus-independent bias".

These data reinforce the notion that the manner in which painful stimuli are presented can profoundly influence how pain is reported. Pain report is not simply dependent upon the subject, but involves an interaction between the subject, experimenter, and paradigm.

\subsection{What is the relevance of this difference?}

This question is complicated by the fact that pain is a multidimensional sensation. One measure may detect more of the sensory components of pain (i.e. intensity, timing, or location) whereas another may be more sensitive to the emotional or affective dimension. Indeed different clinical interventions appear to differentially alter the sensory and affective components of pain (for review (Fernandez and Turk, 1992) therefore it seems logical that experimental paradigms may elicit different dimensions of the pain sensation as well.

Evidence suggests that the random protocol is less biased by affective and evaluative factors such as subject hypervigilance or expectancy, and stimulus-independent bias (Gracely et al., 2003). In contrast, ascending pain paradigms such as the dolorimeter and tender point count correlate more with a subject's psychological state (Petzke et al., 2003b). Again this may play into the 
"known" or expectant property of the ascending paradigms as compared to the "unknown" random paradigms. Subjects know that the succeeding stimulus will be more intense in the ascending paradigms and therefore may react differently to it. Evidence in support of this hypothesis is presented here, where the relative unpleasantness was significantly more in the ascending paradigms than in the random paradigms for both groups (Fig. 3). This suggests that the ascending paradigm evokes a greater affective magnitude than the random paradigm. This is somewhat counterintuitive, in that as noted above the ratings for both intensity and unpleasantness were higher in the random paradigm than for the ascending, yet only the ascending paradigms are related to measures of mood and distress (Petzke et al., 2003b). These data suggest that other psychological constructs, perhaps related to control, are driving symptom report and lowering of pain ratings obtained during ascending paradigms, and that these constructs affect both healthy controls and patients with fibromyalgia in a similar manner.

\section{Conclusion}

The dimensions of pain intensity and unpleasantness are separate yet highly correlated. Results presented here further support this hypothesis and in addition suggest that the presence of a chronic pain state can alter one's response to evoked pain. Indeed Rollman reasoned that chronic pain subjects may display a different "adaptation-level" than normal subjects based on differences in their "internal comparison" mechanisms of pain stimuli (Rollman, 1979). As noted above FM subjects may compare the experimental pain to their more intense clinical pain and therefore rate the evoked sensations as less unpleasant.

Results presented here are consistent with those obtained from other chronic pain states such as cancer and causalgia (Price et al., 1987). In general clinical pain is perceived as more unpleasant than experimentally evoked sensations of similar magnitude. This raises the hypothesis that differing chronic pain conditions may elicit similar changes in evoked pain perceptions, however uncovering the underlying mechanisms will require further research.

\section{Acknowledgements}

FP was supported by a grant of the Deutsche Forschungsgemeinschaft (Pe 713/1-1). Analysis of this work was supported by Department of the Army Grant DAMD 17-00-2-0018 and NIH award 1-K01AT01111-01.

\section{References}

Bajaj P, Arendt-Nielsen L, Madsen H. Sensory changes during the ovulatory phase of the menstrual cycle in healthy women. Eur J Pain 2001;5:135-44.

Bajaj P, Madsen H, Arendt-Nielsen L. A comparison of modalityspecific somatosensory changes during menstruation in dysmenorrheic and nondysmenorrheic women. Clin J Pain 2002;18:180-90.

Bendtsen L, Norregaard J, Jensen R, Olesen J. Evidence of qualitatively altered nociception in patients with fibromyalgia. Arthritis Rheumatol 1997;40:98-102.

Boissevain MD, McCain GA. Toward an integrated understanding of fibromyalgia syndrome. II. Psychological and phenomenological aspects. Pain 1991;45:239-48.

Burckhardt CS, O'Reilly CA, Wiens AN, Clark SR, Campbell SM, Bennett RM. Assessing depression in fibromyalgia patients. Arthritis Care Res 1994;7:35-9.

Derogatis LR, Spencer PM, The Brief Symptom Inventory: Administration, Scoring, and Procedures Manual-I, Clinical Psychometric Research, Baltimore, 1983.

Eliav E, Gracely RH. Sensory changes in the territory of the lingual and inferior alveolar nerves following lower third molar extraction. Pain 1998;77:191-9.

Epstein SA, Kay G, Clauw D, Heaton R, Klein D, Krupp L, et al. Psychiatric disorders in patients with fibromyalgia: a multicenter investigation., San Diego, 1997.

Fernandez E, Turk DC. Sensory and affective components of pain: separation and synthesis. Psychol Bull 1992;112:205-17.

Fields HL. Pain: an unpleasant topic. Pain 1999(Suppl. 6):S61-9.

Geisser ME, Casey KL, Brucksch CB, Ribbens CM, Appleton BB, Crofford LJ. Perception of noxious and innocuous heat stimulation among healthy women and women with fibromyalgia: association with mood, somatic focus, and catastrophizing. Pain 2003;102:243-50.

Gracely RH. Evaluation of multi-dimensional pain scales. Pain 1992;48:297-300.

Gracely RH. Studies of pain in human subjects. In: Wall PD, Melzack R, editors. Textbook of pain. London: Churchill Livingstone; 1999. p. $385-407$.

Gracely RH, Dubner R, McGrath PA. Narcotic analgesia: fentanyl reduces the intensity but not the unpleasantness of painful tooth pulp sensations. Science 1979;203:1261-3.

Gracely RH, McGrath F, Dubner R. Ratio scales of sensory and affective verbal pain descriptors. Pain 1978a;5:5-18.

Gracely RH, McGrath P, Dubner R. Validity and sensitivity of ratio scales of sensory and affective verbal pain descriptors: manipulation of affect by diazepam. Pain 1978b;5:19-29.

Gracely RH, Petzke F, Wolf JM, Clauw DJ. Functional magnetic resonance imaging evidence of augmented pain processing in fibromyalgia. Arthritis Rheumatol 2002;46:1333-43.

Gracely RH, Grant MA, Giesecke T. Evoked pain measures in fibromyalgia. Best Pract Res Clin Rheumatol 2003;17:593-609.

Hofbauer RK, Rainville P, Duncan GH, Bushnell MC. Cortical representation of the sensory dimension of pain. J Neurophysiol 2001;86:402-11.

Keogh E, Mansoor L. Investigating the effects of anxiety sensitivity and coping on the perception of cold pressor pain in healthy women. Eur J Pain 2001;5:11-22.

Kosek E, Ekholm J, Hansson P. Modulation of pressure pain thresholds during and following isometric contraction in patients with fibromyalgia and in healthy controls. Pain 1996;64:415-23.

Lautenbacher S, Rollman GB. Possible deficiencies of pain modulation in fibromyalgia. Clin J Pain 1997;13:189-96.

Lembo T, Naliboff BD, Matin K, Munakata J, Parker RA, Gracely $\mathrm{RH}$, et al. Irritable bowel syndrome patients show altered sensitivity to exogenous opioids. Pain 2000;87:137-47. 
Malow RM. The effects of induced anxiety on pain perception: a signal detection analysis. Pain 1981;11:397-405.

McDermid AJ, Rollman GB, McCain GA. Generalized hypervigilance in fibromyalgia: evidence of perceptual amplification. Pain 1996;66:133-44.

Melzack R. The short-form McGill pain questionnaire. Pain 1987;30:191-7.

Melzack R, Casey KL. Sensory, motivational, and central control determinants of pain: A new conceptual model. In: Kenshalo DR, editor. The skin senses. Springfield, IL: Charles C. Thomas; 1968. p. $423-39$.

Miron D, Duncan GH, Bushnell MC. Effects of attention on the intensity and unpleasantness of thermal pain. Pain 1989;39:345-52.

Petzke F, Khine A, Williams D, Groner K, Clauw DJ, Gracely RH. Dolorimetry performed at 3 paired tender points highly predicts overall tenderness. J Rheumatol 2001;28:2568-9.

Petzke F, Clauw DJ, Ambrose K, Khine A, Gracely RH. Increased pain sensitivity in fibromyalgia: effects of stimulus type and mode of presentation. Pain 2003;105:403-13.

Petzke F, Gracely RH, Park KM, Ambrose K, Clauw DJ. What do tender points measure? Influence of distress on 4 measures of tenderness. J Rheumatol 2003;30:567-74.

Price DD. Psychological and neural mechanisms of the affective dimension of pain. Science 2000;288:1769-72.

Price DD, Barrell JJ, Gracely RH. A psychophysical analysis of experimental factors that selectively influence the affective dimension of pain. Pain 1980;8:137-49.

Price DD, Rafii A, Watkins LR, Buckingham B. A psychophysical analysis of acupuncture analgesia. Pain 1984;19:27-42.

Price DD, Harkins SW, Baker C. Sensory-affective relationships among different types of clinical and experimental pain. Pain 1987;28:297-307.

Rainville P, Feine JS, Bushnell MC, Duncan GH. A psychophysical comparison of sensory and affective responses to four modalities of experimental pain. Somatosens Mot Res 1992;9:265-77.

Rainville P, Duncan GH, Price DD, Carrier B, Bushnell MC. Pain affect encoded in human anterior cingulate but not somatosensory cortex. Science 1997;277:968-71.
Riley JL, Zawacki TM, Robinson ME, Geisser ME. Empirical test of the factor structure of the West Haven Yale Multidimensional Pain Inventory. Clin J Pain 1999;15:24-30.

Rollman GB. Signal detection theory pain measures: empirical validation studies and adaptation-level effects. Pain 1979;6:9-21.

Rollman GB. Measurement of experimental pain in chronic pain patients: methodological and individual factors. In: Melzack R, editor. Pain measurement and assessment. New York: Raven Press; 1983. p. 251-8.

Sawamoto N, Honda M, Okada T, Hanakawa T, Kanda M, Fukuyama $\mathrm{H}$, et al. Expectation of pain enhances responses to nonpainful somatosensory stimulation in the anterior cingulate cortex and parietal operculum/posterior insula: an event-related functional magnetic resonance imaging study. J Neurosci 2000;20:7438-45.

Staud R, Robinson ME, Vierck Jr CJ, Price DD. Diffuse noxious inhibitory controls (DNIC) attenuate temporal summation of second pain in normal males but not in normal females or fibromyalgia patients. Pain 2003;101:167-74.

Sternberg WF, Bailin D, Grant M, Gracely RH. Competition alters the perception of noxious stimuli in male and female athletes. Pain 1998;76:231-8.

Stohler CS, Kowalski CJ. Spatial and temporal summation of sensory and affective dimensions of deep somatic pain. Pain 1999;79: 165-73.

Ware JEJ, Kosinski M. Interpreting SF-36 summary Health measures: a response. Qual Life Res 2001;10:405-13.

Ware JEJ, Snow K, Kosinski M, Gandek B. SF 36 Health Survey Manual and Interpretation Guide. Lincoln: Quality Metric, Inc.; 2000, $320 \mathrm{pp}$.

Wolfe F. The relation between tender points and fibromyalgia symptom variables: evidence that fibromyalgia is not a discrete disorder in the clinic. Ann Rheumatol Dis 1997;56:268-71.

Wolfe F, Smythe HA, Yunus MB, Bennett RM, Bombardier C, Goldenberg DL, et al. The American College of Rheumatology 1990 Criteria for the Classification of Fibromyalgia. Report of the Multicenter Criteria Committee. Arthritis Rheumatol 1990;33:160-72. 